\title{
El rastreo poblacional con mamografía disminuye la mortalidad por cáncer de mama pero incrementa el riesgo de sobrediagnóstico y sobretratamiento
}

Population screening mammography reduces mortality from breast cancer but increases the risk of overdiagnosis and overtreatment

Gøtzsche P y col. Cochrane Database Syst Rev. 2013;CD001877.

\begin{abstract}
Objetivos
Evaluar, el efecto sobre la mortalidad del tamizaje de cáncer de mama (CM) mediante mamografía en mujeres de 39 a 74 años de edad sin dicho diagnóstico previo.
\end{abstract}

\section{Diseño y fuente de datos}

Revisión sistemática y meta-análisis de ensayos clínicos aleatorizados que evaluaran tamizaje mamográfico vs. no tamizaje. Se buscó en Pubmed y en la plataforma internacional de ensayos clínicos de la Organización Mundial de la Salud (hasta noviembre de 2012).

\section{Selección de estudios y extracción de datos}

Dos autores independientes evaluaron lo ensayos a incluir. Extrajeron datos sobre el número de personas aleatorizadas, el tipo de mamografía, el número y la intensidad de los tamizajes, así como la mortalidad por CM, por cualquier cáncer y la mortalidad global, evaluando también el número de intervenciones quirúrgicas y de la implementación de otras terapias adyuvantes.

\section{Resultados principales}

Fueron identificados ocho estudios elegibles. Uno fue excluido porque la aleatorización no produjo grupos comparables. Los siete estudios elegibles incluyeron un total de 600.000 mujeres. Los resultados principales fueron divididos de acuerdo a calidad del proceso de aleatorización. Ver tabla 1.

Los autores estiman que por cada 2.000 mujeres tamizadas durante diez años se evitará una muerte por $\mathrm{CM}$, diez mujeres serán sobrediagnosticadas y tratadas innecesariamente, y más de 200 experimentarán ansiedad por un resultado falsamente positivo en el tamizaje.

Tabla 1: eficacia del tamizaje con mamografía vs. no tamizaje durante 13 años de seguimiento.

\begin{tabular}{|c|c|c|c|c|c|c|c|}
\hline \multirow{2}{*}{\multicolumn{3}{|c|}{ Subanálisis }} & \multicolumn{2}{|c|}{ Muertes por cáncer mamario } & \multirow{2}{*}{$\begin{array}{l}\text { Riesgo relativo } \\
\text { (IC95\%) }\end{array}$} & \multirow{3}{*}{$\begin{array}{c}\text { NNR } \\
\text { (IC95\%) }\end{array}$} & \multirow[t]{2}{*}{$\mathbf{p}$} \\
\hline & & & \multirow{2}{*}{$\begin{array}{c}\text { Tamizaje } \\
\text { n/total (\%) } \\
404 / 119.504 \\
0,338 \% \\
\end{array}$} & \multirow{2}{*}{$\begin{array}{c}\text { No tamizaje } \\
\text { n/total (\%) } \\
572 / 172.649 \\
0,331 \% \\
\end{array}$} & & & \\
\hline $\begin{array}{l}\text { Mortalidad } \\
\text { por }\end{array}$ & $\begin{array}{l}\text { De acuerdo } \\
\text { a la calidad }\end{array}$ & Adecuada & & & $\begin{array}{c}0,9 \\
(0,79 \mathrm{a} 1,02)\end{array}$ & & 0,10 \\
\hline $\begin{array}{l}\text { cáncer } \\
\text { mamario }\end{array}$ & de la aleatorización & Sub-optima & $\begin{array}{c}633 / 170.048 \\
0,372 \%\end{array}$ & $\begin{array}{c}746 / 136.889 \\
0,545 \%\end{array}$ & $\begin{array}{c}0,75 \\
(0,67 \mathrm{a} 0,83)\end{array}$ & 578 & $<0,00001$ \\
\hline & $\begin{array}{c}\text { Por } \\
\text { grupo }\end{array}$ & Menores de 50 años & $\begin{array}{c}385 / 142.906 \\
0,269 \%\end{array}$ & $\begin{array}{c}567 / 186.605 \\
0,304 \%\end{array}$ & $\begin{array}{c}0,84 \\
(0,73 \mathrm{a} 0,96)\end{array}$ & 2.857 & 0,0085 \\
\hline & etario & Mayores de 50 años & $\begin{array}{c}599 / 146.284 \\
0,409 \%\end{array}$ & $\begin{array}{c}701 / 122.590 \\
0,572 \% \\
\end{array}$ & $\begin{array}{c}0.77 \\
(0,69 \mathrm{a} 0,86) \\
\end{array}$ & 613 & $<0,00001$ \\
\hline \multicolumn{3}{|c|}{ Número de mastectomías y lumpectomías } & $\begin{array}{c}2.405 / 145.536 \\
1,652 \%\end{array}$ & $\begin{array}{c}1419 / 104.943 \\
1,352 \%\end{array}$ & $\begin{array}{c}1,35 \\
(1,26 \mathrm{a} 1,44)\end{array}$ & & $<0,00001$ \\
\hline \multicolumn{3}{|c|}{ Mortalidad total a 13 años } & $\begin{array}{c}4.644 / 119.897 \\
3,87 \%\end{array}$ & $\begin{array}{c}5.671 / 173.061 \\
3,28 \%\end{array}$ & $\begin{array}{c}0,99 \\
(0,95 \mathrm{a} 1,03)\end{array}$ & & 0,63 \\
\hline
\end{tabular}

NNR: número necesario a rastrear.

\section{Conclusiones}

Se asume que el tamizaje reduce la mortalidad por CM en $15 \%$ y que incrementa el riesgo de sobrediagnóstico y sobretratamiento en $30 \%$.
Fuente de financiamiento/conflicto de interés de los autores: Rigshospitalet, Denmark. Danish Institute for Health Technology Assessment, Denmark. La extracción de datos fue independiente y los autores declararon no tener conflictos de interés.

\section{Comentario}

El CM es uno de los canceres más frecuentes ${ }^{1}$ y uno de los más publicitados en los medios de comunicación, lo que probablemente contribuya a que sea uno de los que más temor produce en las mujeres, y por el cual estas demanden la realización de mamografías a edades cada vez más tempranas (p. ej. a los 40 años). En nuestro esfuerzo por proteger a nuestros pacientes debemos considerar los beneficios y los riesgos potenciales de las diferentes pruebas de rastreo y compartirlos con ellos para que puedan tomar una decisión informada. Respecto del CM, conviene diferenciar el concepto de mamografía diagnóstica -la que se hace luego de la detección de un nódulo en la mama-, del de mamografía de tamiza$\mathrm{je}^{2}$-la que se hace en una mujer en quien no existen razones para pensar que pueda existir un riesgo especial de padecer $\mathrm{CM}$. El artículo que estamos comentando está referido a este último. La comunidad sobreestima los beneficios de la mamografía y no suele tener conocimientos sobre los riesgos ${ }^{3}$-el sobrediagnóstico y el sobretratamiento-, que pueden resultar como consecuencia de los programas de tamizaje de $\mathrm{CM}$. Vale destacar que en mujeres menores de 50 años éste riesgo es mucho mayor y el beneficio, escaso (1/2.857) o nulo, especialmente si solo consideramos los estudios de mejor calidad metodológica. Para quienes día a día nos responsabilizamos del cuidado de personas, es importante reevaluar el beneficio real del tamizaje poblacional con mamografía. Como nos informa el trabajo que acabamos de resumir, si solo se analizan los estudios con mejor calidad metodológica (tres de los siete ensayos analizados por el grupo Cochrane Nórdico), no se encuentra beneficio. Por el contrario, cuando analizamos la totalidad de los estudios identificados, la probabilidad de sufrir daño por sobrediagnóstico y/o sobretratamiento es diez veces mayor que la de beneficiarse del rastreo.

\section{Conclusiones del comentador}

No existe evidencia sólida que avale el beneficio del tamizaje poblacional de CM mediante mamografía ya que los riesgos de sobrediagnóstico y sobretratamiento son altos. Esta información debería ser ofrecida en el momento de ofrecer la mamografía de tamizaje y debería ser temada en cuenta por quienes elaboran políticas públicas. 
María Sofía Cuba Fuentes [ Universidad Peruana Cayetano Heredia. maria.cuba@upch.pe ]

Luis Aguilera García [ Facultad de Medicina, Universidad de Valladolid, España. Wonca Iberoamericana-CIMF ]

Cuba Fuentes MS, y Aguilera García L. El rastreo poblacional con mamografía disminuye la mortalidad por cáncer de mama pero incrementa el riesgo de sobrediagnóstico y sobretratamiento. Evid Act Pract Ambul. 2015;18(4):118-119. Oct-Dic. Comentado de: Gøtzsche PC, y col. Screening for breast cancer with mammography. Cochrane Database Syst Rev. 2013;CD001877. PMID: 23737396.

\section{Referencias}

1. Organización Mundial de la Salud. Cáncer de mama: prevención y control [sitio en línea]. Ginebra, Suiza. Disponible en URL: http://www.who.int/topics/cancer/breastcancer/es/index1.html (último acceso, diciembre 2015)

2. Welch $\mathrm{H}$. We look harder for breast cancer. En: Welch HG, Ovediagnosed: making people sick in the pursuit of health. $1^{\text {a }}$ ed. Massachusetts: Beacon Press books; 2011. 3. Biller Andomo N. Abolishing mammography screening programs? A view from the swiss medical board. N Engl J Med. 2014 May 22;370(21):1965-7.

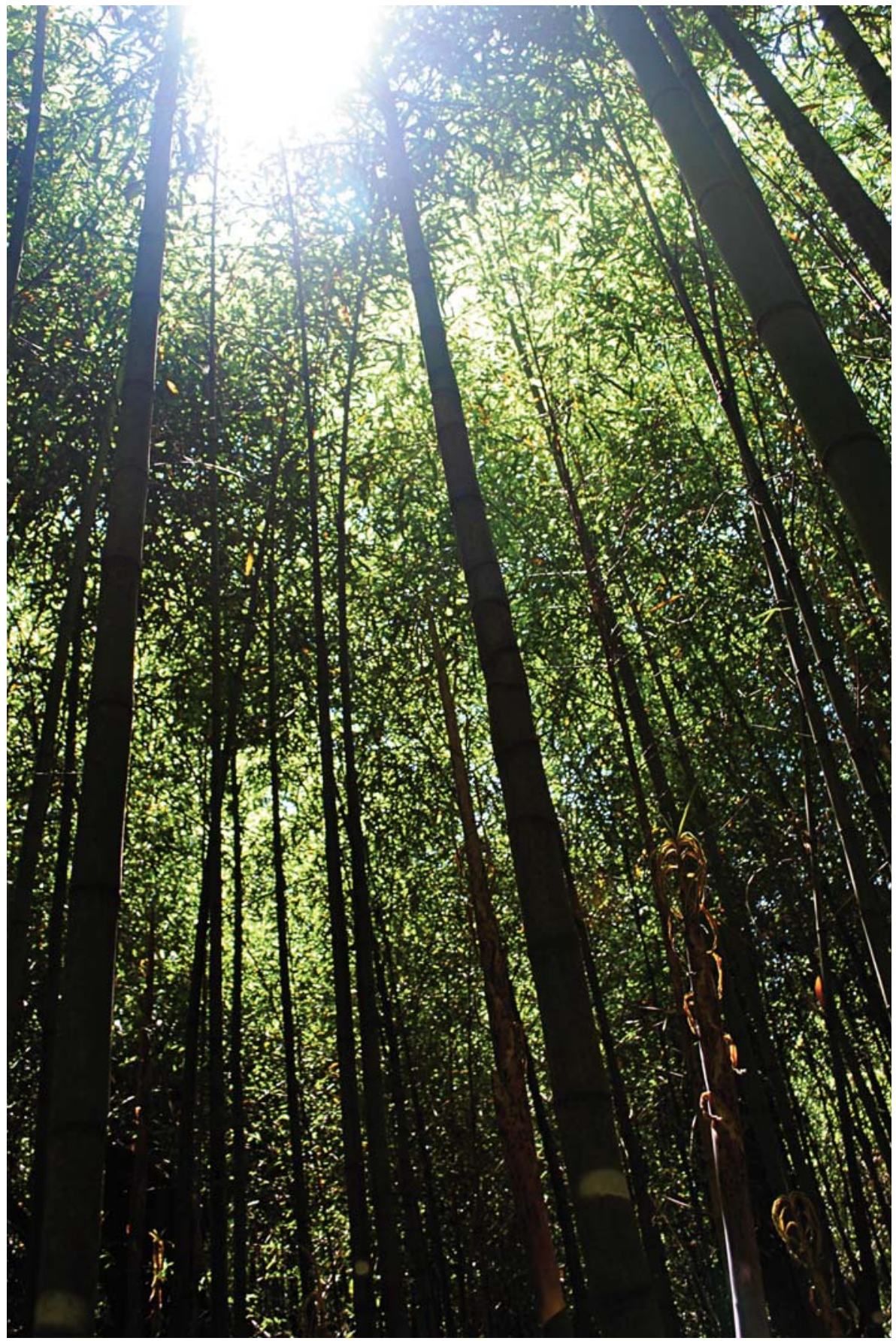

Fotografía: Silvia Spina. 